\title{
Effectiveness of Nutrition/Health Behavior Change Communication Training; Micronutrient Supplementation and Lipid-Nutrient Supplements to Improve Child Health in Western Kenya
}

\author{
John Ombogo ${ }^{1 *}$, Silvenus Konyole ${ }^{2}$, Gordon Nguka ${ }^{2}$, Kelvin Musau ${ }^{1}$ and Marion Kiprotich ${ }^{1}$ \\ ${ }^{1}$ Nutrition Department, Kakamega Head Quarters, Kenya \\ ${ }^{2}$ Nutritional Science Department, Masinde Muliro University of Science and Technology, Kenya \\ *Corresponding author: John Ombogo, Government Relation, Nutrition Department, One Acre Fund, Kakamega Head Quarters, Kenya

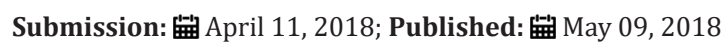

\begin{abstract}
Despite robust economic growth in the recent decades; $48 \%$ of Kenya's children under five years are chronically malnourished; i.e. stunted (see KDHS; 2014; UNICEF). Levels of anemia are increasing with 70 percent of children fewer than five years being affected and Kenya has one of the highest under-5 mortality rates in Sub-Saharan Africa. Nearly half of these deaths (43\%) account to mild and moderate malnutrition; another $11 \%$ are related to severe forms of malnutrition. Stunting in linear growth occurs mainly during the first 1000 days; from conception through 24 months of age. In recognition of this critical period; there have been few evaluations of the growth impact of interventions that cover most of this window. The objective of this study was to evaluate home fortification approaches for preventing maternal and child under nutrition within a community-based health program through Ministry of Health and One Acre Fund Partnership. The research hypothesizes that folic acid and small-quantity lipid-based nutrient supplements (LNS) provided to women during pregnancy and the first 6 months postpartum; LNS provided to their offspring from 6 to24 months of age; or both would result in greater child length-for-age z score (LAZ) at 24 months than iron and folic acid (IFA) provided to women during pregnancy and postpartum plus micronutrient powder (MNP) or no supplementation for their offspring from 6 to 24 months. We conducted a cluster-randomized effectiveness trial with 2 arms: 1) women and children both received LNS (LNS-IFAs group); 2) women received IFAs (IFA group). IFA-group was our control. We enrolled 600 women at 20 weeks of gestation within 10 clusters; each comprising the supervision area of a Community Health Volunteer. Data was collected electronically using Mobile Application and extracted into excel spread sheet for cleaning before analysis. Analyses were primarily performed by using ANCOVA F tests and Tukey-Kramer-corrected pair wise comparisons. Home fortification with small-quantity LNS; during the first 1000 days was analyzed to show any improvement on child linear growth and head size in Siaya County. Infants in the LNS-IFAS group had higher birth weights $(2629 \pm 408$ compared with $2588 \pm 413 \mathrm{~g} ; \mathrm{P}=0.007)$; weight for-age $\mathrm{z}$ scores $(-1.48 \pm 1.01$ compared with $-1.59 \pm 1.02$; $\mathrm{P}=0.006)$; head-circumference-for-age $\mathrm{z}$ scores (HCZs; -1.2661 .08 compared with $-1.34 \pm 1.12 ; \mathrm{P}=0.028)$; and body mass index z scores $(-1.57 \pm 1.05$ compared with $-1.66 \pm 1.03 ; \mathrm{P}=0.005$ ) than those in the IFA group; in adjusted models; the differences in length ( $47.6 \pm 0.07$ compared with $47.4 \pm 0.04 \mathrm{~cm} ; \mathrm{P}=0.043$ ) and LAZ $(-1.15 \pm 0.04$ compared with $-1.24 \pm 0.02 ; \mathrm{P}=0.035)$ were also significant. LNS-IFAs reduced the risk of newborn stunting $(18.7 \%$ compared with 22.6\%; RR: 0.83; 95\% CI: 0.71; 0.97) and small head size (HCZ <-2) (20.7\% compared with 24.9\%; RR: 0.85; 95\% CI: 0.73; 0.98). The effects of LNS-IFA on newborn stunting were greatest in infants born before a 10-weeks interruption in LNS-IFA distribution ( $\mathrm{n}=137 ; 15.7 \%$ compared with 23.6\%; adjusted RR: $0.69 ; 95 \%$ CI: $0.53 ; 0.89$ ) and in infants born to women $\leq 24 \mathrm{y}$ of age or with household food insecurity. Prenatal lipid-based nutrient supplements can improve birth outcomes in Kenyan women; especially those at higher risk of fetal growth restriction
\end{abstract}

Keywords: Iron and folic acid; Lipid-based nutrient supplements; Head circumference; Mid upper hand circumference; Low birth weight; Newborn stunting; Birth outcomes; Effectiveness

\section{Introduction}

Poor linear growth of children is a major problem in developing countries; where an estimated $28 \%$ of children < $5 y$ of age are stunted [1]. Childhood stunting is associated with several negative consequences; including impaired cognitive development [2] and increased risk of death from infectious diseases [3]. Although stunted children may experience catch-up growth; their mean height deficit usually persists into adulthood [4]; a situation that is linked to low productivity and increased risk of non-communicable diseases. Because of the deleterious short- and long-term consequences of stunting; its prevention has become a key national and international priority [5]. 
The process of stunting is most marked during the first 12-18 months [6] of life; but it usually begins in utero or soon after birth and may continue until 24 months of age [7]. It is logical; therefore; that interventions to reduce childhood stunting may need to include both the prenatal period and the first 1-2 postnatal years. In a previous landmark study by the Institute of Nutrition of Central America and Panama conducted in the 1970s; a comprehensive approach involving supplementation during pregnancy; lactation; and early childhood was associated with greater length gain in children and long-term effects on cognitive development and adult stature. However; since the Institute of Nutrition of Central America and Panama trial; there have been very few efforts to evaluate the impact of comprehensive nutritional supplementation given during most of the "first 1000 days" on stunting.

The International Lipid-Based Nutrient Supplements (iLiNS) Project developed small-quantity $(20 \mathrm{~g} / \mathrm{d}$ or $118 \mathrm{kcal} / \mathrm{d})$; lipidbased nutrient supplements (SQ-LNSs) for pregnant and lactating women and for infants [8]; following initial work with infants in Ghana and Malawi; with the purpose of enriching local diets with micronutrients and essential fatty acids (EFAs). SQ-LNSs were designed to address concerns that in many populations the total energy content of the usual diet may be adequate; but the micronutrient and EFA contents may be low. In the iLi NS-DYAD trials carried out in Ghana and Malawi by the International Lipidbased Nutrient Supplement study group; in which mother-child dyads were enrolled; the efficacy of SQ-LNSs provided to women during pregnancy and the first 6 months postpartum and to their offspring from 6 to 18 months of age was tested by using a similar study design. In Malawi; this supplementation strategy may have had a modest impact on birth length among selected subgroups of women [9]; but it did not affect child growth by 18 months of age when compared with 2 control groups; namely iron and folic acid (IFA) provided during pregnancy only or a multiple micronutrient (MMN) supplement with most of the same micronutrients as the SQ-LNSs provided during pregnancy and the first 6 months postpartum. For the Ghana study; we previously reported on the first of the 2 primary hypotheses [10] and showed that infants born to mothers who received prenatal supplementation with SQ-LNSs compared with IFA had higher birth weight; and among those born to primiparous women; infants also had higher birth length and head circumference than both control groups (IFA and MMN). This research will report on the second hypothesis; namely that child growth at 18 months of age would be greater in children whose mothers received SQ-LNSs and who themselves were supplemented with SQ-LNSs than in those whose mothers were in the control groups.

\section{Background}

Stunting remains a big public health problem in Kenya. Thirtyseven percent of fewer than five children are stunted in Western Kenya. While at global level there has been a significant reduction in stunting levels; currently at 27\%; Kenya has failed to register significant progress towards reducing stunting. One of the major factors contributing to high levels of stunting in Western Kenya is suboptimal infant and young child feeding practices; including poor breastfeeding and dietary practices. So far evidence is lacking on the contribution of the amount of breast milk on infant and child growth and development

after 6 months of exclusive breastfeeding. The present study will have four aims; to assess the effect of small quantity lipid-based nutrient supplement (SQ-LNS) on child development; to assess energy and nutrient in take among pregnant women supplemented with SQ-LNS and those in the control group; to assess determinants of adherence and finally to assess the relationship between SQ-LNS and growth and development among infants and young children age 9-18 months in Siaya County.

\section{Problem statement}

As the latest National Demographic Health Survey revealed; child malnutrition in Western Kenya was still alarmingly high in 2014. Four percent (4\%) of children below 5 years were wasted; $11 \%$ underweight and even $26 \%$ stunted (KDHS 2014). More recent data on the development of child malnutrition in Kenya is currently unavailable and urgently required to monitor the development and take necessary action. The American development agency USAID stresses that evidence on the effectiveness of rehabilitation approaches for malnourished children in Kenya; including micronutrient supplementation and interventions that foster behavioral change; is vital for scaling up effective interventions and combating child malnutrition. Also the Kenya Nutritionists and Dietitians Institute assume nutrition supplements and nutrition trainings to be key drivers towards decreased levels of child malnutrition in the country. KNDI through its regular workshops also pointed out that there is a knowledge gap concerning nutrition interventions for malnourished children in Kenya. Moreover; the scientists stressed that there is a lack of studies which investigate the effectiveness of interventions aiming at improving the quality of home diets; this will be included in the nutrition/health education intervention group of this research project.

\section{Justification}

LNS are currently being used in programs targeting pregnant women in developing countries with the expectation of improving birth outcomes and reducing LBW [11,12] and current studies have shown mixed effects of this intervention using varying composition; dose; frequency and comparison groups between studies. This study assessed the effects and safety of LNS for women during pregnancy on maternal; birth and infant outcomes; as currently there is no systematic evaluation in this domain; and attempted to assess the appropriate composition; frequency and duration of this intervention through various subgroup analyses. In addition; we carried out a subgroup analysis on whether the pregnant women who were identified and the LNS to be distributed through a facility or in a community. We also developed a companion review to assess the effectiveness of preventive lipid-based nutrient supplements (LNS) given with complementary foods on health; nutrition and developmental outcomes of non-hospitalized infants and children 6 to 23 months of age (Das in press). These studies were to guide policy makers to make informed decisions on the effectiveness and safety of LNS compared to other interventions and also 
assessed which delivery platforms are effective. Micronutrient supplements and powders are not recommended for pregnant women; based on the lack of evidence for their impact on maternal anaemia [13]; but they were compared to the provision of LNS; given the assumption that other micronutrients contained in the micronutrient powders could have an impact on pregnancy; birth and infant developmental outcomes; for example; zinc on preterm births [14]. We also compared LNS to antenatal nutrition education; since nutrition education conducted during the antenatal period with the aim of increasing energy and protein intake appears to be effective in reducing the risk of preterm birth and LBW; increasing head circumference at birth; increasing birth weight among undernourished women; and increasing protein intake [15]

\section{Objectives}

1. To determine factors affecting adherence to LNS intake.

2. To review the effects of lipid-based nutrient supplements (LNS) when given to women during pregnancy; and their impact on maternal; birth and infant outcomes.

3. To relate the Birth Weights of newborns in the treatment and control arms on quality of birth outcome.

4. To determine the association between LNS intake and birth outcomes among pregnant women.

\section{Scope of the study}

This study investigated how to meet the nutritional requirements of pregnant women most effectively through nutritional package and behavior change communication trainings. A comparative intervention study showed a positive LNS supplementation; nutrition trainings are suited boosting maternal health. A special focus was laid upon pregnancy; as the first 1000 days from perception of a child to the completion of the second year are vital to prevent chronic malnutrition with all its consequences - like retarded brain development; limited learning capacity; increased morbidity and child mortality rates [16]. A costeffectiveness analysis was to allow formulating recommendations for the County Government to learn from this study and scale up effective measures on a broader scale to decrease child mortality.

\section{Hypotheses}

1. Mothers with better knowledge on nutrition/health issues cook improved family meals

and are less likely to have malnourished children. The nutrition education/health intervention group shall verify or rather falsify this assumption.

2. Pregnant women who cover their nutritional needs are less likely to have low birth weight children.

The nutrition supplementation groups were to proof these two latter assumptions. There were two interventional subgroups; one providing a commercial supplement (e.g. "LNS") to; one who will only receive non-nutritional items respectively [17].

\section{Materials and Methods}

\section{Study design overview}

In order to obtain a comprehensive picture about the current nutritional situation of pregnant women at Siaya County a baseline survey was conducted. Afterwards; a comparative intervention study was to shed light on the question if intensive nutrition/health trainings; LNS supplementation can strengthen the growth and health development of women and children below 3 years [18]. Finally; this research project was to give an insight how the Siaya County Government could overcome continuing high levels of child malnutrition. A cluster sampling was applied for the base line survey with a total coverage of $n=630$ households. For the intervention study; out of the researched areas of the baseline survey; up to 5 clusters were selected where the same or a comparative prevalence of moderate stunting; served as primary indicator of the study; was given-this was to guarantee comparability of results on the basis of a similar stunting prevalence at the onset of the intervention. Under these conditions; all four intervention groups were to use the same control group to achieve synergies. While IFA-control and LNS-IFAsnutrition supplementation groups were in geographic nearness; the nutrition/health education intervention group located at an adequate distance to prevent dismissing of training contents and the danger of resulting biases. Each intervention group was to cover at least 8 pregnant women [19-23].

\section{Baseline survey}

The baseline survey was to assess the local food/nutrition security and vulnerability situation. The following fields will be of primary interest:

1. The share of women with low MUAC in pregnancy

2. The prevalence of anemia in women and children in the survey area.

3. The share of newborn children with low birth weight

4. The pregnant women knowledge on nutritious food; health and hygiene

5. The HH's food intakes (diversity of diets)

6. The HH's capacity to purchase food items from the markets (purchasing power)

7. The HH's access to markets; health facilities and water/ sanitation facilities

\section{Study site}

The research area was Siaya County in Alego Usonga; Gem and Ugenya Sub-Counties; covering a total of $\mathrm{N}=175$; 490 inhabitants. Clusters comprising an equal number of inhabitants were defined according to locally available statistic data. Thereafter; 15 clusters were randomly selected to conduct the baseline survey targeting at a cluster sample size of 42 households $(\mathrm{HH})$ with children less than 3 years. A total sample size of $630 \mathrm{HH}$ was covered altogether $[24,25]$. 


\section{Study participants}

All households with pregnant women were eligible. All mothers were invited to participate in the survey and were accepted if they agreed for anthropometric measurements and blood samples be taken.

\section{Sample size baseline survey}

The local prevalence of moderately stunted children under three years around Siaya is estimated to amount to 39 percent (estimate based on own measurements). Assuming a sampling error d of 0.04 and applying a confidence level of 95 percent $(\mathrm{z}=1.96)$; a minimal sample size of 150 households is necessary to conduct the baseline survey [26].

\section{Statistical methods and analysis}

Data analysis was done with the statistical software SPSS. This was to help to determine basic descriptive analyses such as counts and percentages. Respectively; the exact prevalence of moderate stunting around Siaya can be determined and it can be tested whether girls are more likely to be stunted than boys via cross tabulation testing [27].

\section{Risks and benefits}

There were no obvious risks involved for the participants. Benefits comprise the following:

1. Knowledge gain about the nutritional/health situation of the family.

2. Offer to visit any facility in Siaya County; where treatment is possible free of costs any time for children and mothers.

\section{Data collection}

The baseline data was collected in 3 steps:

1. Directly at the Household - anthropometric data; laboratory examination

a. Draw blood of mothers to determine hemoglobin ( $\mathrm{Hb})$

b. Take weight and MUAC of pregnant mothers

c. Take Head Circumference; Weight and Length of Newborns.

\section{Nearby the LNS-IFAs-interviews}

a. Overall nutritional situation (e.g. socio-economic situation; food security/diversity)

b. Nutrition knowledge (e.g. healthy food; hygiene)

3. At home with the family-weight protocol of consumed food

The investigator was to weigh the actual amounts eaten by pregnant women and to understand the nutritional gap of the primary target group [28]. This intervention was time consuming and the investigator needed to stay one whole day in each $\mathrm{HH}$. Yet; it is a necessary action; as 24-hour-recalls are less likely to give the actual amounts consumed (merely the items-not the real quantities-might is remembered). The sample size was limited to $\mathrm{n}=30$ pregnant women. The results will help to calculate how much LNS has to be supplemented to cover the RDA of iron; and other micronutrients.

\section{Intervention study}

The intervention study was to help to understand the role of nutrition/health education;

lipid nutrient supplementation and behavior change communication in pregnant women via the nutrition intervention by One Acre Fund through the Ministry of Health $[29,30]$.

For this purpose; different groups-treatment and control-were defined:

1. LNS-IFAs Group where mothers were trained in nutrition; hygiene and health and received LNS

2. IFA Group without treatment (control group)-pregnant women who received non-food items

The baseline study was to give decisive insights to optimize the design of the intervention study. The following 3 links were made:

1. confirmation of the sample size;

2. curriculum of the nutrition/health training intervention; and

3. specification of the amount of LNS given

Moreover; the baseline survey was to give an overall understanding of the nutrition situation in the survey area; which is currently missing due to a lack of recent data.

\section{Study sites}

Five (5) clusters surveyed in the baseline study with equal prevalence of moderate stunting (e.g. 40\%) were randomly selected as study sites of the intervention study. This was to guarantee comparable results for the interventions and control group-stunting was defined as primary indicator as most studies so far have been focusing on wasting and stunting is the most spread form of malnutrition in Siaya. Yet; the nutrition education group needed to have the same stunting prevalence but be geographically separated to prevent spreading of teaching contents and finally biased study results. For each intervention group; LNS-IFAs group needed to be randomly selected within the given cluster.

\section{Participants}

All pregnant women coming to the LNS-IFAs group were to take food and enjoy the respective treatment; LNS and nutrition education and wash behavior change communication. The LNSIFAs group received LNS until the child is 6 months of age. This was to allow to see longtime effects of the treatments and to determine the defaulter rate. To be included into the data evaluation process; pregnant woman needed to agree that anthropometric measurements and blood samples will be taken repeatedly. All severely malnourished women (MUAC $<28.5 \mathrm{~cm}$ ) were referred to Siaya Health Facilities for intensive nutrition therapy and medical treatment as required. This service was free of cost. 


\section{Sample size calculation and justification}

The change in prevalence of moderate stunting among children aged 6-36 months was to serve as primary indicator for the intervention study. A reduction of $15 \%$ in moderate stunting was detectable. At the outset; $39 \%$ of children were estimated to suffer from moderate stunting ( $\mathrm{P} 1=0.39)$. Hence; the target level of moderate stunting was $24 \%(\mathrm{P} 2=0.24)$. A power of $80 \%$ is and a significance level of $95 \%$ ( $Z \beta=0.840$ and $Z \alpha=1.645)$ is chosen. $n=$ $(\mathrm{Z} \alpha+\mathrm{Z} \beta) 2 *((\mathrm{P} 1 *(1-\mathrm{P} 1)+\mathrm{P} 2 *(1-\mathrm{P} 2)) /(\mathrm{P} 2-\mathrm{P} 1) 2=(1.645+0.840) 2$ $*((0.39 * 0.61)+(0.24 * 0.76)) /(0.24-0.39) 2=116$ pregnant women Adding a 20\% allowance for non-response; a sample size of $n=150$ children was targeted (each for the treatment and control groups).

\section{Statistical methods and analysis}

Again; the statistical analysis was done with the software SPSS. It was determined; if growth; health and motor development of the children in the treatment groups differ significantly from the children' health and growth development as well as motor capacities in the control group. After testing the data for normal distribution; t-tests were applied to analyze potential differences and recognize the effectiveness of the interventions. To prove the posed hypotheses; one was to check several points through statistical analysis:

i. Do infants from women on LNS-IFAs group; recover more quickly from stunting than control group infants?

ii. Do infants from women on LNS-IFAs group and under nutrition education/BCC get sick less frequently than the control group infants?

iii. Do infants from women on LNS-IFAs group and under nutrition education/BCC have better motor capacities than control group infants?

\section{Risks and benefits}

One risk in supplementing pregnant women was that problems under delivery might occur when the head of the newborn baby becomes too big for the still slim mother with narrow pelvis. Yet; as the pregnant women are connected to the Siaya Health Facilities; antenatal checkups including sonography were to allow recognizing difficult deliveries beforehand. It was then possible to refer pregnant women with need of a caesarean delivery to nearby nursing homes and the costs were beard by the project holder. Normal deliveries were conducted free of cost at the Siaya County Health Facilities [31].

Benefits for the participants included the following:

i. Provision of LNS until the child is 2 years (for nutrition supplementation groups)

ii. Nutrition Education and WASH BCC training for two years targeted at different gestation age.

iii. Free Referral Service and Treatment at Siaya health Facilities where treatment is possible free of costs any time for infants and mothers. iv. The control group will get access to the most effective treatment

\section{Data collection}

A set of indicators were applied to assess the growth and health of the infants in the comparative intervention study. Largely quantitative indicators were applied with addition of one qualitative component (mother's opinion-focus group interviews). In order to assess growth and health of the infants under treatment in comparison to the control group children; anthropometric data was gathered and health. Also mothers were to listen how they perceive the health and nutritional situation of their infants. Moreover; adherence was the main issue within the data collection process; as it was obviously influencing the success of all interventions. Thus adherence was low in one treatment group; but high in another; this was seen in the outcomes achieved. It was crucial to understand the reasons for non-adherence and aim to motivate participants to eat the full meal given and attend all nutrition/health trainings offered; respectively [32].

\section{Ethical Considerations}

The decision of the Institutional Ethics Committee of the Masinde Muliro University of Science and Technology concerning the conduct of the study was made in writing to the SponsorInvestigator before commencement of this study. The clinical study began upon approval from both Ethics Committees was received. Any additional requirements imposed by the IERC were implemented to the best possible. Substantial amendments were only implemented following prior approval of the IERC. CHVs were explain to each participant in the study; the nature of the study; its purpose; the procedures involved; the expected duration; the potential risks and benefits and any discomfort it may entail. The information was provided in the language best understood by the participant/caregiver (Swahili and Luo). Each participant was informed that the participation in the study was voluntary and that she was to withdraw herself from the study at any time and that withdrawal of consent was not to affect her subsequent medical assistance and treatment. The participant was informed that her medical records were only examined by authorised individuals other than health care staff if need arises [33,34].

All participants for the study were provided a participant information sheet (in Swahili or Luo) and a consent form describing the study and providing sufficient information for the participant to make an informed decision about their own participation in the study. In case of illiterate participants; the study was explained in the presence of an impartial witness or read out by the impartial witness that is chosen by the potential study participant. Enough time was given to the participants to decide whether to participate or not. This was particularly important as in some circumstances the pregnant woman needed to await her husband's/the head of household's approval for participation.

The participant information sheet and the consent form were submitted to the local IERC for review and approval. The formal consent of a participant; using the approved consent form; was 
obtained before the participant was submitted to any study procedure. The participant read; or have read in case of illiterate participants in the presence of an impartial witness; and consider the statement before signing and dating the informed consent form; and was given a copy of the signed document. The consent was also signed and dated by the investigator (or his designee) and it was retained as part of the study records. Consent was taken from the pregnant woman for herself and her offspring. Participants was not to receive any compensation for their participation but they received baby clothing and blankets as a small incentive (both control and intervention group). The investigator affirmed and upheld the principle of the participant's right to privacy and that they shall comply with applicable privacy laws. Especially; anonymity of the participant's was guaranteed when presenting the data at scientific meetings or publishing them in scientific journals.

Individual subject medical information obtained as a result of this study was considered confidential and disclosure to third parties was prohibited; except in circumstances where medical follow-up was required pursuant to information obtained. Subject confidentiality was further ensured by utilising subject identification code numbers to correspond to treatment data in the computer files. Direct access to source documents was only being permitted to the advisory board members if requested by them. Access to all study related documents (protocol; questionnaires; dataset; randomization code; etc.) during and after the study was managed by the sponsor-investigator who was delegate access to the respective documents.

Data from the study was stored on Comm. Care's secure cloud server. Only selected OAF Kenya Monitoring \& Evaluation staff and the investigator were to have access to the password-protected data on Comm. Care. They were able to access the data as it is uploaded from the tablets. De-identified data was downloaded regularly on password-protected servers for monitoring purposes; which was to serve as a back-up. The serum samples collected for the analysis of micronutrient status was stored until final lab results are received and validated from the Vit Min Lab. Six months after the results were considered as final; the samples were destroyed. The duplicate sample; which were held as back-up samples in Kenya in case something happens to the samples during shipment to Germany; was also be destroyed six months after results are considered final. The investigators had an advisory board that reviewed the protocol; tools and baseline reports; followed by moderated teleconferences to discuss course correction. They also reviewed the final reports.

\section{Quality assurance}

Health Field Officers employed by One Acre Fund collected data using Tablets already installed with Comm. Care and data was exported to Excel Spread Sheet for cleaning before further exported to SPSS. They undertook an intensive training and field practice during the pretest period to sharpen their skills and knowledge on data collection. Community Health Volunteers also underwent training on Nutrition and WASH BCC since they did product delivery and data collection as well

\section{The main selection criteria were:}

1. English and Local language skills (Luo or Swahili preferable)

\section{Open to learn about nutrition and health issues}

3. Ready to continue the work for 24 months.

The investigators were trained about the research process and learnt to fulfill all steps; especially to take anthropometric data. A pretest was done to ensure that the investigators had understood clearly their tasks and quality of data was reliable.

\section{Data protection}

All data sets were encoded via SPSS as to prevent that conclusions about individuals can be drawn. In no publication any individual data will be presented.

\section{Results}

Between 15 October 2016 and 31 March 2018; we screened 400 pregnant women for eligibility and enrolled 300 (150 in the LNSIFAs group and 150 in the IFA group). Although we had anticipated enrolling over a period of 6 months; after 4 months we had already recruited our target of 300 women; so we conserved resources since we stopped enrollment after 5th month. Compared with women who were enrolled; those who were not enrolled because of ineligibility or refusal to consent $(n=100$ were; on average; slightly older $(23.2 \pm 5.4$ compared with $21.9 \pm 5.0$ y; $\mathrm{P}=0.001)$ and less educated ( $5.8 \pm 3.5$ compared with $6.2 \pm 3.2 \mathrm{y}$; $\mathrm{P}=0.03$ ). Loss of pregnancy or stillbirth occurred in $8.5 \%$ of women: $13(8.5 \%)$ in the LNS-IFAs group and $9(6.4 \%)$ in the IFA group. A total of 278 live births took place between 15 January 2018 and 5 March 2018. We had birth anthropometric data for 272 infants; but 6 of them were excluded from the analysis due to late measurement (after $14 \mathrm{~d}$ of birth) or unknown measurement date. Therefore; anthropometric data for 272 infants were analyzed (137 in the LNS-IFAs group and 135in the IFA group). There was no significant difference between groups in the percentage of participants with usable birth anthropometric data of those enrolled $(85.8 \%$ in the LNS-IFAs group compared with $86.1 \%$ in the IFA group; $\mathrm{P}=0.80$ ). Mean ages of the newborns on the day of anthropometric measurement were $2.11 \pm 2.86 \mathrm{~d}$ in the LNS-IFAs group and $2.01 \pm 2.70 \mathrm{~d}$ in the IFA group. Compared with mothers of infants with birth anthropometric data; the mothers of the infants with no anthropometric data were less educated; less wealthy; more likely to be nulliparous; and were enrolled earlier in gestation (data not shown). At baseline; sociodemographic; anthropometric; and obstetric characteristics of pregnant women were similar between LNS-IFAs $(n=137)$ and IFA $(n=141)$ groups; with the exception of "years of formal education" (6.4 \pm 3.2 compared with $6.1 \pm 3.3$ y; $\mathrm{P}=0.023$ ). On average; the women were $22 \mathrm{y}$ of age. Mean maternal height was $151 \mathrm{~cm}$; mean BMI (in $\mathrm{kg} / \mathrm{m} 2$ ) was 20; approximately one third of the women were thin $(\mathrm{BMI}<18.5)$; and $39-42 \%$ was nulliparous. The mean gestational age at enrollment was 13 weeks in both groups. The intervention groups were also balanced when we considered only those women whose infants were included in the birth outcomes 
analyses for this study. On the basis of maternal retrospective recall (at 6 weeks postpartum) of overall adherence throughout pregnancy; the percentages of mothers who reported regular consumption (every day oral most every day) were 64\% in the LNSIFAs group and $92 \%$ in the IFA group ( $\mathrm{P}=0.001)$. This difference in adherence is consistent with additional adherence data collected during pregnancy from a subgroup of participants [35]. Compared with regular adherers; women with lower adherence were taller; younger; more educated; wealthier; and more food secure (data not shown). Infants in the LNS-IFAs and IFA groups differed significantly with respect to birth weight (2629 compared with 2588g); WAZ (-1.48 compared with -1.59); head circumference (32.75 compared with $32.65 \mathrm{~cm})$; HCZ (-1.26compared with -1.34$)$; and BMIZ $(-1.57$ compared with -1.66). Adjustment for predetermined covariates did not change these results. However; after adjustment for covariates; the differences in birth length (47.6 compared with 47.4 $\mathrm{cm})$ and LAZ (-1.15 compared with -1.25$)$ became significant. There was a trend toward a significant difference $(\mathrm{P}<0.10)$ with respect to MUAC. There was no significant effect of LNS provision on duration of gestation. Infants in the LNS-IFAs and IFA groups differed significantly with respect to the prevalence of stunting (LAZ $<-2$; 18.7\% compared with 22.6\%); small head size (HCZ <-2; $20.7 \%$ compared with 24.9\%); low BMI (BMIZ; 22; 30.2\%compared with $34.7 \%$ ); and SGA (63.3\% compared with $67.3 \%$ ). There was a trend toward a significant difference in LBW $36.0 \%$ compared with $39.5 \%)$. There was no significant effect on preterm delivery.

Tests for interactions with potential effect modifiers were not significant $(\mathrm{P}<0.10)$ for maternal education; primiparity; or $\mathrm{BMI}$; but were significant for one or more birth outcomes for household food insecurity; maternal age; height; household assets; child sex; and time of year at birth. Household food insecurity modified the effect of LNS-IFAs on gestational age at delivery; birth length; head circumference; WAZ; LAZ; HCZ; preterm birth; underweight; and stunting. The risk of stunting at birth was reduced by LNS-IFAs (compared with IFA) in those living in households categorized with severe; moderate; or mild food insecurity; whereas the difference was not significant among those living in households categorized as food secure. LNS-IFAS increased the duration of gestation among women in households categorized with severe or moderate food insecurity (but the treatment group differences were not significant within the other2 categories of food security). The same trends were observed for mean birth length and head circumference (i.e.; there was a greater effect of LNS-IFAs in households with higher food insecurity). The distribution of LAZs of the newborns in the LNS-IFA sand IFA groups; within each of the 4 food-insecurity subgroups; suggested that LNS-IFAs reduced the proportion of infants with low LAZs at birth; with little effect on the mean or upper end of the distribution.

Maternal age modified the effect of LNS-IFAs (compared with IFA) on newborn stunting; with larger effects seen among women 14-24 years of age and no significant effect among women $\geq 25$ years of age. There was no between group differences in the percentage of women aged $14-24$ years $(72.6 \%$ in the LNS-IFAs group and $72.3 \%$ in the IFA group). Maternal age was not a significant effect modifier for any of the other birth outcomes. Maternal height modified the effect of LNS-IFAS on the prevalence of LBW (P-interaction=0.065): among women whose height was above the median $(>150.5 \mathrm{~cm})$; there was a significant difference between intervention groups (26.6\% in the LNS-IFAs group and $31.9 \%$ in the IFA group; $\mathrm{P}=0.027$ ); but there was no difference in shorter women $(45.9 \%$ and $47.2 \%$; respectively). Household assets modified the effect of LNS-IFAs on birth length; LAZ; MUAC; and SGA; with group differences (greater birth size in the LNS-IFAs group) seen consistently in the lowest wealth quintile and for some outcomes also in the third wealth quintile. The effect of LNS-IFAs (compared with IFA) on the duration of gestation (P-interaction $=0.054$ ) was significant for female infants (39.6 \pm 0.11 compared with $39.3 \pm 0.06$ weeks; $\mathrm{P}=0.020$ ) but not male infants $(39.3 \pm 0.11$ compared with $39.3 \pm 0.06$ weeks; $\mathrm{P}=0.775$ ). Time of year at birth (in 2-months intervals) was a significant effect modifier for birth weight; length; head circumference; MUAC; WAZ; LAZ; HCZ; LBW; stunting; and low HCZ; but the results did not exhibit a consistent seasonal pattern and are confounded by the fact that the disruption in LNS-IFAS supply occurred during one of those intervals. Exploratory analyses were conducted to examine the influence of the 10-weeks disruption in the supply of LNS-IFAs by comparing the LNS-IFAs and IFA groups within the following 3 subgroups of women: 1) those who delivered before the LNS-IFAs supply disruption; 2) those who delivered during the suspension of LNS-IFAs distribution who were in the last 1-10 weeks of pregnancy at that time and 3) those who delivered after the disruption but who experienced a 10-weeks gap in LNS-IFAs distribution during pregnancy if they had been assigned to the LNSIFAs. The prevalence of newborn stunting was significantly lower in the LNS-IFAs group among infants born before the disruption but not in those born later; the same trend was observed for head circumference. In those who were born before the disruption; LNSIFAs reduced the risk of newborn stunting by $31 \%$ and the risk of small head size by $21 \%$.

Per protocol analyses were consistent with a stronger apparent impact of the intervention on birth outcomes when women with low reported adherence were excluded. For example among women reporting "regular" supplement consumption during pregnancy (but not excluding those affected by the disruption in LNS-IFAs supply); LNS-IFA reduced new born stunting by $25 \%$ and small head size by $19 \%$. There were no significant differences between groups in the incidence of any of the serious adverse events assessed.

\section{Discussion}

In this study; the provision of LNS-IFAs during pregnancy (compared with IFA) significantly increased mean birth weight; WAZ; birth length; LAZ; head circumference; HCZ; and BMIZ. Although the differences in mean birth length and LAZ were small; there was a greater shift at the lower end of the distribution of LAZs; resulting in a significant $17 \%$ reduction in the prevalence of newborn stunting. We also found significant reductions in the prevalence of small head size and low BMI at birth. The per protocol analyses (excluding women with low reported adherence but not 
excluding those affected by the disruption in LNS-IFAs supply) were consistent with the shift being attributable to LNS-IFAs; with a $25 \%$ reduction in newborn stunting among women who reported regularly consuming LNS-IFAS compared with those who reported regularly consuming IFA. Among infants born before the 10 -weeks disruption in supply of LNS-IFAs; the reduction in newborn stunting was $31 \%$. To our knowledge; this is the first study to report an effect of a prenatal nutrient or food supplement containing MMNs on the prevalence of stunting at birth.

Although the proportion of stunting that occurs before rather than after birth is not well understood and likely varies across populations; there is agreement that stunting often begins in utero [1] and some of the stunting that occurs after birth may be programmed in utero [35]. However; meta analyses have not shown any significant impact on birth length of prenatal MMN [36] or balanced protein-energy supplementation [37] despite significant effects on birth weight; although a recent large prenatal MMN trial in Bangladesh reported a small $(0.2 \mathrm{~cm})$ but significant effect on birth length [38]. By contrast; in Burkina Faso; there was a $0.5 \mathrm{~cm}$ increase in birth length (but no difference in birth weight) in the group who received prenatal LNSs(373kcal/d) compared with those who received MMNs [39].

In our cohort; the effect of LNS-IFAs on mean birth weight $(+47$ gin the unadjusted and $46 \mathrm{~g}$ in the adjusted model) was similar to the pooled effect of MMNs in the most recent meta-analysis(+53g) [40] but smaller than the estimated pooled effect of balanced proteinenergy supplementation (+73g) [41]; which is not surprising given that LNS-IFAS contributed only $118 \mathrm{kcal} / \mathrm{d}$ (compared with w400$800 \mathrm{kcal} / \mathrm{d}$ in most prenatal food supplementation studies) and the RDNS was an effectiveness study; not an efficacy trial. There was a trend toward a reduction in LBW (7\%); whereas the abovementioned meta-analyses reported reductions in LBW of $14 \%$ for MMNs and 32\% for balanced protein-energy supplementation. However; in our per protocol analyses; the prevalence of LBW was reduced by $11 \%(\mathrm{P}=0.03)$; which is similar to what has been observed for efficacy trials with MMNs. We also found a $13 \%$ reduction in the prevalence of wasting (low BMI) at birth (a 14\% reduction in per protocol analyses).

The significant effects of LNS-IFAs on head circumference and percentage with small head size at birth are noteworthy given the association between head circumference and brain size during

infancy [42]. The percentage of newborns with a small head size was reduced by $17 \%$ in the sample as a whole; by $19 \%$ in per protocol analyses; and by $22 \%$ when limited to infants born before the 10-weeks disruption in the supply of LNS-IFAs. The MMN supplement meta-analyses mentioned earlier [36] did not report data on head circumference; and some other published MMN [43,44] and LNS [39] studies did not show any significant differences.

However; a recent meta-analysis showed that prenatal MMN supplementation increased child head circumference later in infancy or childhood; compared with the provision of $\$ 2$ micronutrients [45]. In the recent large prenatal MMN intervention in Bangladesh; there was also a significant effect on head circumference [46]. Although the most recent meta-analysis of prenatal MMN supplementation [47] and the latter study in Bangladesh [46] reported a small but significant increase in the duration of gestation; we did not find significant main effects of LNS-PLs on duration of gestation or preterm delivery. However; in subgroup analyses; LNS-IFAs increased the duration of gestation by 0.5 week among women in very-food-insecure households and by 0.3 week among women carrying female infants. There are at least 2 possible explanations for the lack of a main effect on duration of gestation. First; in contrast to the other study in Bangladesh; ours was an effectiveness trial and adherence to LNS-IFAs was significantly lower than adherence to FA. Second; the average duration of gestation at enrollment was 13 weeks (range: 4.6-20.0 weeks); whereas all of the women in the other Bangladesh study were recruited in the first trimester.

We explored whether the magnitude of the effect of LNSIFAs on birth size differed depending on several pre-defined; biologically plausible; potential effect modifiers. There were no significant interactions with maternal education; primiparity; or BMI. However; household food insecurity; household assets; maternal age and height; sex of the child; and time of year at birth modified the effect of LNS-IFAs on at least one birth outcome. Women in food-insecure households are more likely to suffer from both macro and micronutrient deficiencies during pregnancy; therefore; it is not surprising that we found a greater effect of LNS-IFAs among such women; not only on newborn stunting but also on mean birth length and head circumference (as well as duration of gestation; as mentioned above). What is surprising is that the differences in the prevalence of newborn stunting across subgroups with higher levels of household food insecurity (as seen in the IFA group) were eliminated in the LNS-IFAs group; although the supplement provided only $118 \mathrm{kcal} / \mathrm{d}$. As a result; newborn stunting was reduced by $36 \%$ among women who were very food insecure. Similarly; the effect of LNS-IFAs on birth length; MUAC; and SGA was more evident in the lower household wealth quintiles.

Maternal age was also an effect modifier: LNS-IFAs reduced newborn stunting by $21 \%$ among infants born to mothers who were 14-24y of age; with no significant effect observed in older women. Pregnancy during adolescence increases the risk of adverse birth outcomes; poor fetal growth; and infant and maternal morbidity [48]; probably at least partly because of competition for nutrients between the young mother and the growing fetus [46]. LNS-IFAs may reduce this competition by providing extra amounts of both micro and macronutrients. The meta-analysis of MMN supplementation during pregnancy did not report any interaction between supplement type and maternal age [49].

As the first effectiveness study to examine how small-quantity LNS-IFAs affect birth outcomes; this study has several strengths and limitations. Strengths include the following: 1) the use of 2 independent teams; one to conduct the intervention (led by LAMB) and another to evaluate impact (led by ICDDR; B and UC Davis); 2) 
enrollment of 300 women who were representative of the target population; 3 ) a low rate of attrition(mostly due to travel out of the study area rather than refusal to participate); 4) use of well-trained anthropometrists who performed measurements according to WHO standards and were standardized; and 5) completion of $86 \%$ of the newborn anthropometric measurements within $72 \mathrm{~h}$ of delivery. Among the limitations; the disruption of the LNS-IFAs supply for a period of10weeks; which was beyond our control; compromised our ability to investigate the full potential of LNSIFAs as an intervention [50-56].

For several key outcomes; we found a larger effect of LNS-IFAs among infants born to women who gave birth before the disruption of LNS-IFAs distribution. We believe that this is consistent with a causal effect of LNS-IFAs on birth size; and thus actually strengthens our conclusions. Second; it was not possible to blind the women to the type of supplement provided; because the supplements were very different in appearance and taste. Nonetheless; researchers responsible for the collection of outcome data were kept blind to study assignment. Third; we used LMP to estimate the duration of gestation; because it was not feasible to use ultrasonography for all participants. Fourth; were lied on the women's reported consumption of the supplements to assess adherence; instead of direct observation; so the adherence data could be inaccurate. Finally; we examined effects within several different targeted subgroups; and these exploratory effect modification results need to be interpreted with caution because we examined multiple hypotheses and the study was not powered to test each potential interaction [57-62]. We conclude that LNS-IFAs supplementation during pregnancy reduced newborn stunting; wasting; and small head size in the study population $[63,64]$. These effects occurred without a significant impact on duration of gestation; which suggests that LNS-IFAs reduce fetal growth restriction but not preterm delivery. As a whole; the study women were at high risk of fetal growth restriction; given that approximately one-third of them had a low BMI and 39\% were $\leq 20$ years of age. A reduction in newborn stunting by LNS-IFAs was most evident among younger women and those residing in households that experienced high levels of food insecurity. Because this was an effectiveness study conducted in the context of an operating community health program; the findings should be relevant to other programs that serve similar populations $[65,66]$.

\section{References}

1. De Onis M, Onyango AW, Vanden Broeck J, Chumlea WC, Martorell R (2004) Measurement and standardization protocols for anthropometry used in the construction of a new international growth reference. Food Nutr Bull 25(1 Suppl): S27-S36.

2. Das JK, Salam RA, Kumar R, Bhutta ZA (2013) Micronutrient fortification of food and its impact on woman and child health: a systematic review. Syst Rev 2: 67.

3. Suchdev PS, Ruth LJ, Woodruff BA, Mbakaya C, Mandava U, et al. (2012) Selling sprinkles micronutrient powder reduces anemia, iron deficiency, and vitamin A deficiency in young children in Western Kenya: a clusterrandomized controlled trial. Am J Clin Nutr 95(5): 1223-1230.

4. Martorell R, Zongrone A (2012) Intergenerational influences on child growth and undernutrition. Paediatr Perinat Epidemiol 26(Suppl 1): 302-314.

5. WHO (2001) Iron Deficiency Anaemia: Assessment, prevention and control; a guide for programme managers. World Health Organization, Geneva, Switzerland.

6. De Regil LM, Suchdev PS, Vist GE, Walleser S, Pena Rosas JP (2011) Home fortification of foods with multiple micronutrient powders for health and nutrition in children under two years of age. Cochrane Database Syst Rev.

7. De Onis M, Dewey KG, Borghi E, Onyango AW, Blossner M, et al. (2013) The World Health Organization's global target for reducing childhood stunting by 2025: rationale and proposed actions. Matern Child Nutr 9(Suppl 2): 6-26.

8. Ashorn P, Alho L, Ashorn U, Cheung YB, Dewey KG, et al. (2015) The impact of lipid-based nutrient supplement provision to pregnant women on newborn size in rural Malawi: a randomized controlled trial. Am J Clin Nutr 101(2): 387-397.

9. Adu Afarwuah S, Lartey A, Okronipa H, Ashorn P, Zeilani M, et al. (2015) Lipid-based nutrient supplement increases the birth size of infants of primiparous women in Ghana. Am J Clin Nutr 101(4): 835-846.

10. Adu Afarwuah S, Lartey A, Okronipa H, Ashorn P, Peerson JM, et al. (2016) Small-quantity, lipid-based nutrient supplements provided to women during pregnancy and 6 mo postpartum and to their infants from $6 \mathrm{mo}$ of age increase the mean attained length of 18-mo-old children in semiurban Ghana: a randomized controlled trial. Am J Clin Nutr 104(3): 797808.

11. Bhutta ZA, Nelson EA, Lee WS, Tarr PI, Zablah R, et al. (2008) Recent advances and evidence gaps in persistent diarrhea. J Pediatr Gastroenterol Nutr 47(2): 260-265.

12. WHO (2017) Guideline: Preventive chemotherapy to control soiltransmitted helminth infections in at-risk population groups. World Health Organization, Geneva, Switzerland.

13. WHO (2017) World Health Statistics 2017: Monitoring health for the SDGs, Sustainable Development Goals. World Health Organization, Geneva, Switzerland.

14. Glinz D, Hurrell RF, Ouattara M, Zimmermann MB, Brittenham GM, et al. (2015) The effect of iron-fortified complementary food and intermittent preventive treatment of malaria on anaemia in 12- to 36-month-old children: a cluster-randomised controlled trial. Malar Journal 14: 347.

15. Hess SY, Abbeddou S, Jimenez EY, Some JW, Vosti SA, et al. (2015) Smallquantity lipid-based nutrient supplements, regardless of their zinc content, increase growth and reduce the prevalence of stunting and wasting in young burkinabe children: a cluster-randomized trial. PLoS One 10(3): e0122242.

16. Dewey KG, Adu Afarwuah S (2008) Systematic review of the efficacy and effectiveness of complementary feeding interventions in developing countries. Matern Child Nutr 4 (Suppl 1): 24-85.

17. Abbeddou S, Yakes Jimenez E, Some JW, Ouedraogo JB, Brown KH, et al. (2017) Small-quantity lipid-based nutrient supplements containing different amounts of zinc along with diarrhea and malaria treatment increase iron and vitamin A status and reduce anemia prevalence, but do not affect zinc status in young Burkinabe children: a cluster-randomized trial. BMC Pediatr 17(1): 46.

18. Curtis V, Cairncross S (2003) Effect of washing hands with soap on diarrhoea risk in the community: a systematic review. Lancet Infect Dis 3(5): 275-281.

19. Dewey KG, Mridha MK, Matias SL, Arnold CD, Cummins JR, et al. (2017) Lipid-based nutrient supplementation in the first $1000 \mathrm{~d}$ improves child growth in Bangladesh: a cluster-randomized effectiveness trial. Am J Clin Nutr 105(4): 944-957. 
20. DHS (2015) Kenya Demographic and Health Survey 2014: Kenya National Bureau of Statistics, Ministry of Health, Nationla AIDS Control Council Kenya Medical Research Institute, National Council for Population and Development, The DHS Program, ICF International.

21. Disease GBD, Injury I, Prevalence C (2016) Global, regional, and national incidence, prevalence, and years lived with disability for 310 diseases and injuries, 1990-2015: a systematic analysis for the Global Burden of Disease Study 2015. Lancet 388(10053): 1545-1602.

22. Elliott AM, Ndibazza J, Mpairwe H, Muhangi L, Webb EL, et al. (2011) Treatment with anthelminthics during pregnancy: what gains and what risks for the mother and child? Parasitology 138(12): 1499-1507.

23. Fall CH, Fisher DJ, Osmond C, Margetts BM (2009) Multiple micronutrient supplementation during pregnancy in low-income countries: a metaanalysis of effects on birth size and length of gestation. Food Nutr Bull 30(4 Suppl): S533-S546.

24. Dangour AD, Watson L, Cumming O, Boisson S, Che Y, et al. (2013) Interventions to improve water quality and supply, sanitation and hygiene practices, and their effects on the nutritional status of children. Cochrane Database Syst Rev.

25. De Regil LM, Suchdev PS, Vist GE, Walleser S, Pena Rosas JP (2011) Home fortification of foods with multiple micronutrient powders for health and nutrition in children under two years of age. Cochrane Database Syst Rev.

26. Development Initiatives (2017) Global Nutrition Report 2017: Nourishing the SDGs. Bristol, England, UK.

27. $1 \mathrm{AF}$ (2016) One acre fund-annual report. One Acre Fund.

28. FAO (2015) FAO Statistical Pocketbook 2015: World food and agriculture. Food and Agriculture Organization of the United Nations.

29. Food and Nutrition Board, Institute of Medicine (2001) Dietary Reference Intakes for Vitamin A, Vitamin K, Arsenic, Boron, Chromium, Copper Iodine, Iron, Manganese, Molybdenum, Nickel, Silicon, Vanadium, and Zinc: The National Academic Press, Washington, USA.

30. Food and Nutrition Board, Institute of Medicine (2005) Dietary Reference Intakes for Energy, Carbohydrate, Fiber, Fat, Fatty Acids, Cholesterol, Protein, and Amino Acids: The National Academic Press, Washington, USA

31. King JC (2003) The risk of maternal nutritional depletion and poor outcomes increases in early or closely spaced pregnancies. J Nutr 133(5 Suppl 2): 1732S-1736S.

32. Lorenz JM, Whitaker AH, Feldman JF, Yudkin PL, Shen S, et al. (2009) Indices of body and brain size at birth and at the age of 2 years: relations to cognitive outcome at the age of 16 years in low birth weight infants. ] Dev Behav Pediatr 30(6): 535-543.

33. Macharia Mutie CW, Moretti D, Vanden Briel N, Omusundi AM, Mwangi AM, et al. (2012) Maize porridge enriched with a micronutrient powder containing low-dose iron as NaFeEDTA but not amaranth grain flour reduces anemia and iron deficiency in Kenyan preschool children. J Nutr 142(9): 1756-1763.

34. Matias SL, Vargas Vasquez A, Bado Perez R, Alcazar Valdivia L, Aquino Vivanco 0, et al. (2017) Effects of lipid-based nutrient supplements v. micronutrient powders on nutritional and developmental outcomes among Peruvian infants. Public Health Nutr 20(16): 2998-3007.

35. Huybregts L, Roberfroid D, Lanou H, Menten J, Meda N, et al. (2009) Prenatal food supplementation fortified with multiple micronutrients increases birth length: a randomized controlled trial in rural Burkina Faso. Am J Clin Nutr 90(6): 1593-1600.

36. MoH (2011) Kenya National Micronutrient Survey (KNMS).Ministry of Health, UNICEF, USA.

37. Fanzo J (2014) Strengthening the engagement of food and health systems to improve nutrition security: Synthesis and overview of approaches to address malnutrition. Global Food Security 3(3-4): 183-192.

38. Lazzerini M, Wanzira H (2016) Oral zinc for treating diarrhoea in children. Cochrane Database Syst Rev 6: CD005436.

39. WHO (2005) The treatment of diarrhoea: a manual for physicians and other senior health workers. World Health Organization.

40. Imdad A, Bhutta ZA (2012) Maternal nutrition and birth outcomes: effect of balanced protein-energy supplementation. Paediatr Perinat Epidemiol 26(Suppl 1): 178-190.

41. Rohner F, Zimmermann MB, Amon RJ, Vounatsou P, Tschannen AB, et al. (2010) In a randomized controlled trial of iron fortification, anthelmintic treatment, and intermittent preventive treatment of malaria for anemia control in Ivorian children, only anthelmintic treatment shows modest benefit. J Nutr 140(3): 635-641.

42. Imdad A, Yakoob MY, Bhutta ZA (2011) Impact of maternal education about complementary feeding and provision of complementary foods on child growth in developing countries. BMC Public Health 11(Suppl 3): S25.

43. Lu WP, Lu MS, Li ZH, Zhang CX (2014) Effects of multimicronutrient supplementation during pregnancy on postnatal growth of children under 5 years of age: a meta-analysis of randomized controlled trials. PLoS One 9(2): e88496.

44. Bhutta ZA, Rizvi A, Raza F, Hotwani S, Zaidi S, et al. (2009) A comparative evaluation of multiple micronutrient and iron-folic acid supplementation during pregnancy in Pakistan: impact on pregnancy outcomes. Food Nutr Bull 30(4 Suppl): S496-S505.

45. UNICEF (2016) Multiple Micronutrient Powder Supply \& Market Outlook. United Nations Children's Fund, New York, USA.

46. Lundeen E, Schueth T, Toktobaev N, Zlotkin S, Hyder SM, et al. (2010) Daily use of Sprinkles micronutrient powder for 2 months reduces anemia among children 6 to 36 months of age in the Kyrgyz Republic: a cluster-randomized trial. Food Nutr Bull 31(3): 446-460.

47. Kramer MS, Kakuma R (2003) Energy and protein intake in pregnancy. Cochrane Database Syst Rev 4: CD000032.

48. Locks LM, Reerink I, Tucker Brown A, Gnegne S, Ramalanjaona N, et al. (2017) the impact of integrated infant and young child feeding and micronutrient powder intervention on feeding practices and anemia in children aged 6-23 months in madagascar. Nutrients 9(6).

49. Rah JH, De Pee S, Kraemer K, Steiger G, Bloem MW, et al. (2012) Program experience with micronutrient powders and current evidence. J Nutr 142(1): 191S-196S.

50. Kassebaum NJ, Collaborators GBDA (2016) The Global Burden of Anemia. Hematol Oncol Clin North Am 30(2): 247-308.

51. Olveda RM, Acosta LP, Tallo V, Baltazar PI, Lesiguez JL, et al. (2016) Efficacy and safety of praziquantel for the treatment of human schistosomiasis during pregnancy: a phase 2, randomised, double-blind, placebo-controlled trial. Lancet Infect Dis 16(2): 199-208.

52. Petry N, Olofin I, Boy E, Donahue Angel M, Rohner F (2016) The Effect of Low Dose Iron and Zinc Intake on Child Micronutrient Status and Development during the First 1000 Days of Life: A Systematic Review and Meta-Analysis. Nutrients 8(12)

53. Rah JH, Christian P, Shamim AA, Arju UT, Labrique AB, et al. (2008) Pregnancy and lactation hinder growth and nutritional status of adolescent girls in rural Bangladesh. J Nutr 138(8): 1505-1511.

54. Ramakrishnan U, Grant FK, Goldenberg T, Bui V, Imdad A, et al. (2012) Effect of multiple micronutrient supplementation on pregnancy and infant outcomes: a systematic review. Paediatr Perinat Epidemiol 26(Suppl 1): 153-167. 
55. Salam RA, Haider BA, Humayun Q, Bhutta ZA (2015) Effect of administration of antihelminthics for soil-transmitted helminths during pregnancy. Cochrane Database Syst Rev.

56. Salam RA, MacPhail C, Das JK, Bhutta ZA (2013) Effectiveness of micronutrient powders (MNP) in women and children. BMC Public Health 13(Suppl 3): S22.

57. Senbanjo IO, Olayiwola IO, Afolabi WA, Senbanjo OC (2013) Maternal and child under-nutrition in rural and urban communities of Lagos state, Nigeria: the relationship and risk factors. BMC Res Notes 6: 286.

58. Strunz EC, Addiss DG, Stocks ME, Ogden S, Utzinger J, et al. (2014) Water, sanitation, hygiene, and soil-transmitted helminth infection: a systematic review and meta-analysis. PLoS Med 11(3): e1001620.

59. Sunawanq, Utomo B, Hidayat A, Kusharisupeni, Subarkah (2009) Preventing low birth weight through maternal multiple micronutrient supplementation: a cluster-randomized, controlled trial in Indramayu, West Java. Food Nutr Bull 30(4 Suppl): S488-S495.

60. Webb P, Kennedy E (2014) Impacts of agriculture on nutrition: nature of the evidence and research gaps. Food Nutr Bull 35(1): 126-132.

61. Wegmuller R, Camara F, Zimmermann MB, Adou P, Hurrell RF (2006) Salt dual-fortified with iodine and micronized ground ferric pyrophosphate affects iron status but not hemoglobin in children in Cote d'Ivoire. J Nutr 136(7): 1814-1820.

62. West KP, Shamim AA, Mehra S, Labrique AB, Ali H, et al. (2014) Effect of maternal multiple micronutrient vs iron-folic acid supplementation on infant mortality and adverse birth outcomes in rural Bangladesh: the JiVitA-3 randomized trial. JAMA 312(24): 2649-2658.

63. WHO (2007) Preventing and controlling micronutrient deficiencies in populations affected by an emergency. World Health Organisation, World Food Programme, UNICEF.

64. WHO (2016) WHO guideline: Use of multiple micronutrient powders for point- of- use fortification of foods consumed by infants and young children aged 6- 23 months and children aged 2-12 years. World Health Organization, Geneva, Switzerland.

65. WHO/UNICEF (2004) Clinical management of acute diarrhoea. World Health Organization, UNICEF.

66. Woodruff BA, Wirth JP, Bailes A, Matji J, Timmer A, et al. (2017) Determinants of stunting reduction in Ethiopia 2000- 2011. Matern Child Nutr 13(2).
Creative Commons Attribution 4.0

International License

For possible submissions Click Here

\section{Submit Article}

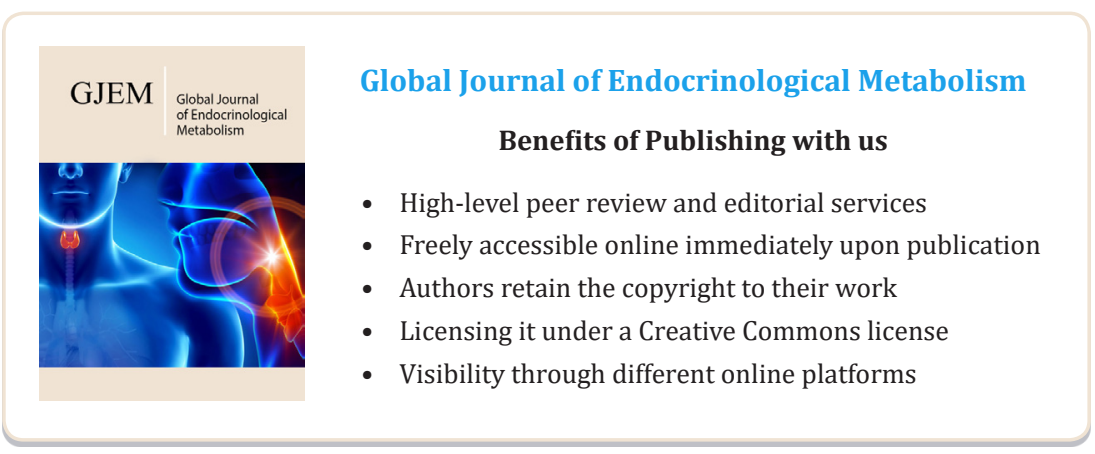

